\title{
Pyruvatdehydrogenase-Defizienz bei einem Kind mit persistierender Lactatacidose
}

\author{
Von E. Dworzak, H. Grunicke \\ Institut für Medizinische Chemie und Biochemie der Universität Innsbruck \\ H. Berger, E. Jarosch, H. Haas \\ Universitätskinderklinik Innsbruck und
}

I. Höpfel

Pathologisches Institut der Universität Innsbruck

(Eingegangen am 27. Oktober 1983/19. Dezember 1984)

Herrn Professor Dr. Erich Kaiser zum 60. Geburtstag gewidmet

Zusammenfassung: Es wird über den Fall einer defekten Pyruvatdehydrogenase (EC 4.1.1.1) in Leukocyten und im Muskelgewebe eines 10-jährigen Knaben mit persistierender Lactatacidose berichtet, der durch Myasthenie und Minderwuchs auffällig wurde. Der Defekt zeigt sich in vitro durch eine verminderte Aktivierbarkeit des Enzyms mit exogener Phosphoproteinphosphatase, in vivo durch eine insuffiziente Stimulierbarkeit des Enzyms bei Muskelarbeit im Vergleich zu gesunden Kontrollen. Die mangelnde Pyruvatdehydrogenaseaktivität erklärt die beobachtete Akkumulierung von Pyruvat, Lactat, Oxalacetat und Alanin und die Verminderung der Konzentration von Citrat im Serum.

Elektronenmikroskopisch können im Muskelgewebe stark vermehrte, vergrößerte Mitochondrien nachgewiesen werden, die auffallend dicht gepackte Cristae besitzen.

Die spontane Pyruvatdehydrogenaseaktivität bei motorischer Ruhe, gemessen ohne Zusatz von Phosphoproteinphosphatase, ist nicht verändert. Es handelt sich somit um eine neue, bisher nicht beschriebene Form einer Pyruvatdehydrogenasedefizienz.

\section{Pyruvate dehydrogenase deficiency in a child with persistent lactic acidosis}

Summary: Evidence is presented for defective pyruvate dehydrogenase (EC 4.1.1.1) in leukocytes and muscle tissue from a 10-year old child with persistent lactic acidosis, suffering from myasthenia and growth retardation. The defect is expressed in vitro by a depressed stimulation of pyruvate dehydrogenase catalytic activity by exogenous phosphoprotein phosphatase, and in vivo by a lack of response to muscle work, in comparison with healthy controls.

Pyruvate dehydrogenase activity is in the normal range when measured without addition of phosphoprotein phosphatase in cells obtained from the resting patient. The defect reported here represents a new, hitherto undescribed form of a pyruvate dehydrogenase deficiency. The insufficient catalytic activity explains the observed accumulation of pyruvate, lactate, oxaloacetate and alanine and the decrease of citrate concentration in the blood of this patient. Electron microscope studies of the muscle tissue show an enhanced number of enlarged mitochondria with bizarre shapes and high densities of cristae. 


\section{Einführung}

Mehrere Fälle einer kongenitalen Lactatacidose wurden bisher beschrieben, die mit Myasthenie, neurologischen Symptomen verschiedenen Ausmaßes und geistiger Retardierung einhergehen (1-4). Die Patienten weisen erhöhte Lactat-, Pyruvat- und Alaninkonzentrationen im Plasma bzw. im Vollblut auf.

Bei einigen Fällen konnten Defekte der Enzyme der Gluconeogenese gezeigt werden $(5-11)$, in anderen Fällen wurde aufgrund der biochemischen Daten eine Verminderung der Pyruvatoxidation für das Krankheitsbild verantwortlich gemacht $(12,13)$.

Nur bei wenigen Fällen war es bislang jedoch möglich, einen Defekt der Pyruvatdehydrogenase oder deren Regulation nachzuweisen $(14,15)$.

Im Unterschied zu den meisten beschriebenen zeigt der vorliegende Fall eine unveränderte Aktivität der nativen Pyruvatdehydrogenase in Rohextrakten. Die Aktivierbarkeit des Enzyms durch Inkubation mit Phosphoproteinphosphatase in vitro sowie durch motorische Aktivität in vivo ist jedoch drastisch reduziert.

Die mangelnde Aktivierbarkeit durch exogene Phosphoproteinphosphatase schließt auch die von Robinson \& Sherwood (21) beschriebene Phosphatase-Defizienz als Ursache für das vorliegende Krankheitsbild aus. Es handelt sich somit um eine neue, bislang nicht bekannte Form einer PyruvatdehydrogenaseDefizienz, deren molekulare Grundlagen diskutiert werden.

\section{Methoden und Material}

\section{Fallbericht}

Ein 10-jähriger Knabe, S. T., zweieiiger Zwilling, fiel im Alter von 7 Jahren nach einer hochfiebrigen Erkrankung durch verminderte körperliche Leistungsfähigkeit, Belastungsdyspnoe und Minderwuchs auf. Im EKG fanden sich Zeichen einer biventrikulären Hypertrophie, ein Vitium cordis konnte nach Herzkatheterisierung ausgeschlossen werden. Der neurologische Status war zunächst unauffällig, EEG und EMG entsprachen der Norm. In letzter Zeit stellen sich epileptiforme Krampfzustände ein; die Intelligenz ist durchschnittlich.

Die Sauerstoffsättigung im peripher entnommenen venösen Blut entsprach mit $72 \%$ der Norm, das arterielle $\mathrm{pO}_{2}$ betrug $45,1 \mathrm{~mm} \mathrm{Hg}$. Die katalytischen Konzentrationen der Enzyme Aspartat- und Alaninaminotransferase, Lactatdehydrogenase sowie Kreatinkinase im Blutserum lagen im Bereich der Norm. Die Glucose wurde nüchtern und nach oraler Belastung mit $38 \mathrm{~g}$ Glucose auf $150 \mathrm{ml}$ Tee nach $30,60,90$ und 120 Minuten im peripher entnommenen venösen Blut bestimmt. Ein 12 stündiger Fastentest unter Bettruhe des Patienten wurde durchgeführt und die Glucose im Plasma nüchtern, nach 2 Stunden, 7 Stunden und nach 12 Stunden bestimmt. Auffallend war die Steigerung von Lactat und Alanin im Blutplasma und von Pyruvat im sofort enteiweißten Vollblut.
Die Konzentration von Citrat im Blutserum war deutlich erniedrigt. Bei einer durchgeführten Arbeitsbelastung des Patienten kam es zu einem pH-Abfall, gleichzeitig sank das Standardbicarbonat. Pyruvat- und Lactatkonzentration sind nach körperlicher Arbeit noch etwa um das 3-fache angestiegen.

Isolierung der Leukocyten und Bereitung des Homogenates

Blutbild des Patienten S. T.: Hb $127 \mathrm{~g} / 1$, Hämatokrit 0,38, Leukocyten $4,4 \cdot 10^{\circ} / 1$, davon $8 \%$ neutrophil stabkernige und $46 \%$ neutrophil segmentkernige Granulocyten, $42 \%$ Lymphocyten und $4 \%$ Monocyten. Die Isolierung der Leukocyten von Patient und gesunden Kontrollen erfolgte nach Blass èt al. (22) aus $30 \mathrm{ml}$ venösem Blut, $1+1$ mit $38 \mathrm{~g} / \mathrm{l} \mathrm{Na}$-Citrat versetzt. Das Leukocytenkonzentrat wurde durch hypotone Lyse der Erythrocyten weitgehend frei von Kontamination gewonnen. Die Zellzahl wurde nach Herstellung einer Zellsuspension in phosphatgepufferter, isotoner $\mathrm{NaCl}-\mathrm{Lösung}$ mikroskopisch bestimmt. Die Zellen wurden in 3 Vol. kaltem $20 \mathrm{mmol} / 1$ Phosphatpuffer $\mathrm{pH}$ 7,0 mit einem Volumenanteil an Glycerin von 0,40 suspendiert und anschließend einmal in flüssigem Stickstoff eingefroren. Sie wurden nach langsamem Auftauen mit dem Omnimix-Desintegrator im Eisbad 2 mal 60 s homogenisiert.

\section{Elektronenmikroskopische Untersuchungen}

Ein Gewebszylinder wurde nach inträoperativer Entnahme aus $\operatorname{dem}$ M. quadriceps femoris auf ein Stäbchen ligiert und in mäßig gestrecktem Zustand sofort in gepuffertem Glutaralde-

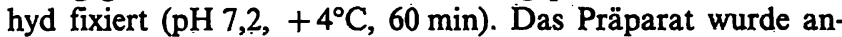
schließend mit 4-maligem Wechsel von Phosphatpuffer ( $\mathrm{pH} 7,2,+4^{\circ} \mathrm{C}, 24 \mathrm{~h}$ ) ausgewaschen. Die Entwässerung erfolgte in aufsteigender Acetonreihe, die Einbettung in Durcopan. Die Semidünnschnitte wurden mit basischem Fuchsin und Methylenblau eingefärbt. Kontrastierung der Dünnschnitte (Reichert OM II) erfolgte mit Uranylacetat $(60 \mathrm{~min})$ und Bleiacetat ( 5 min). Untersuchung und Aufnahme im Elektronenmikroskop Zeiss EM $9 \mathrm{~S}$.

\section{Enzymbestimmung}

Die Pyruvatdehydrogenase-Aktivität wurde nach dem von Schweet et al. (23) und Scriba et al. (24) beschriebenen Verfahren in der Modifikation von Wieland et al. (25) gemessen. Dabei wird das entstehende Acetyl-CoA mit $p$-Nitroanilin in Gegenwart einer Arylaminacetyltransferase zu $p$-Nitroacetanilid umgesetzt und der Umsatz durch Messung der Absorptionsabnahme bei $405 \mathrm{~nm}$ quantitativ bestimmt.

Der Testansatz beinhaltet in einem Endvolumen von $1 \mathrm{ml}$, $25 \mathrm{mmol} / \mathrm{l}$ Tris- $\mathrm{HCl}$ Puffer, pH 8,2, $1 \mathrm{mmol} / 1$-Nitroanilin, $1 \mathrm{mmol} / \mathrm{l} \quad \mathrm{MgCl}_{2}, 20 \mathrm{mmol} / \mathrm{l}$ Mercaptoethanol, $1 \mathrm{mmol} / \mathrm{l}$ $\mathrm{NAD}^{+}, 1 \mathrm{mmol} / \mathrm{l}$ Thiamindiphosphorsäureester, $1 \mathrm{mmol} / \mathrm{l}$ $\mathrm{CoASH}, 20 \mu \mathrm{g}$ Lactatdehydrogenase (Kristallsuspension), 50-100 mU Arylaminacetyltransferase und $200 \mu \mathrm{l}$ Leukocytenhomogenat. Die Reaktion wird durch Zugabe von $100 \mu \mathrm{l}$ $200 \mathrm{mmol} / \mathrm{l} \mathrm{Na}$-Pyruvatlösung gestartet; diese wird zur Ermittlung des Probenleerwertes durch Wässer ersetzt. Zur Bestimmung der dephosphorylierten Form der Pyruvatdehydrogenase wurden $200 \mu \mathrm{l}$ Leukocytenhomogenat mit $50 \mu \mathrm{l} 60 \mathrm{mmol} / \mathrm{l}$ $\mathrm{MgCl}_{2}$-Lösung und $50 \mu \mathrm{l}$ (etwa $400 \mu \mathrm{g}$ ) Schweineherzphosphatase $20 \mathrm{~min}$ bei $25^{\circ} \mathrm{C}$ inkubiert. Die Aktivitätsmessung erfolgt nach oben beschriebenem Ansatz, das Probenvolumen beträgt hier $100 \mu l$.

\section{Reagenzien}

Arylaminacetyltransferase (EC 2.3.1.5) aus Taubenleber wurde nach der Methode von Wieland et al. (26) am Institut für Diabetesforschung, Krankenhaus Schwäbing in München präpariert und uns von Prof. Dr. $O$. Wielaind freundlicherweise zur 
Verfügung gestelit. Aus demselben Institut stammt auch die Phosphoproteinphosphatase aus Schweineherz (27), die uns ebenfalls von Prof. Wieland überlassen wurde.

TPP (Cocarboxylase, Thiamindiphosphorsäureester) bezogen wir von E. Merck, Darmstadt, alle anderen Reagenzien in analytischem Reinheitsgrad stammen von Boehringer Mannheim. Pyruvat und Lactat wurden mit der Testkombination von Boehringer Mannheim nach der Methode von Cloc et al. (28) bzw. nach $F$. Noll (29) bestimt. Citrat wurde nach St. Dagley (30), Oxalacetat nach Hohorst et al. (31) bestimmt.

\section{Resultate}

Schema 1 zeigt ein vereinfachtes Schema zur Darstellung des Enzymdefektes und der Konsequenz einer defekten Pyruvatoxidation.

In Tabelle 1 a sind die klinisch-chemischen Analysen des Patienten bei Bettruhe und nach einer Arbeitsbelastung mit 20 Treppensteigen im Vergleich zu Referenzwerten wiedergegeben. Auffallend sind die erhöhten Werte von Lactat, Pyruvat, Oxalacetat und Alanin und der exzessive Basenüberschuß bereits bei motorischer Ruhe. Gleichzeitig wurde eine deutlich erniedrigte Citratkonzentration im Serum gemessen.

Tabelle $1 \mathrm{~b}$ zeigt die Glucosewerte nüchtern und nach oraler Glucosebelastung und während eines 12-stündigen Fastentests bei Bettruhe. Diese Befunde deuten auf eine Störung der Pyruvatverwertung hin. Eine Lactatacidose als Folge einer Pyruvatcarboxylasedefizienz wurde beschrieben (32).

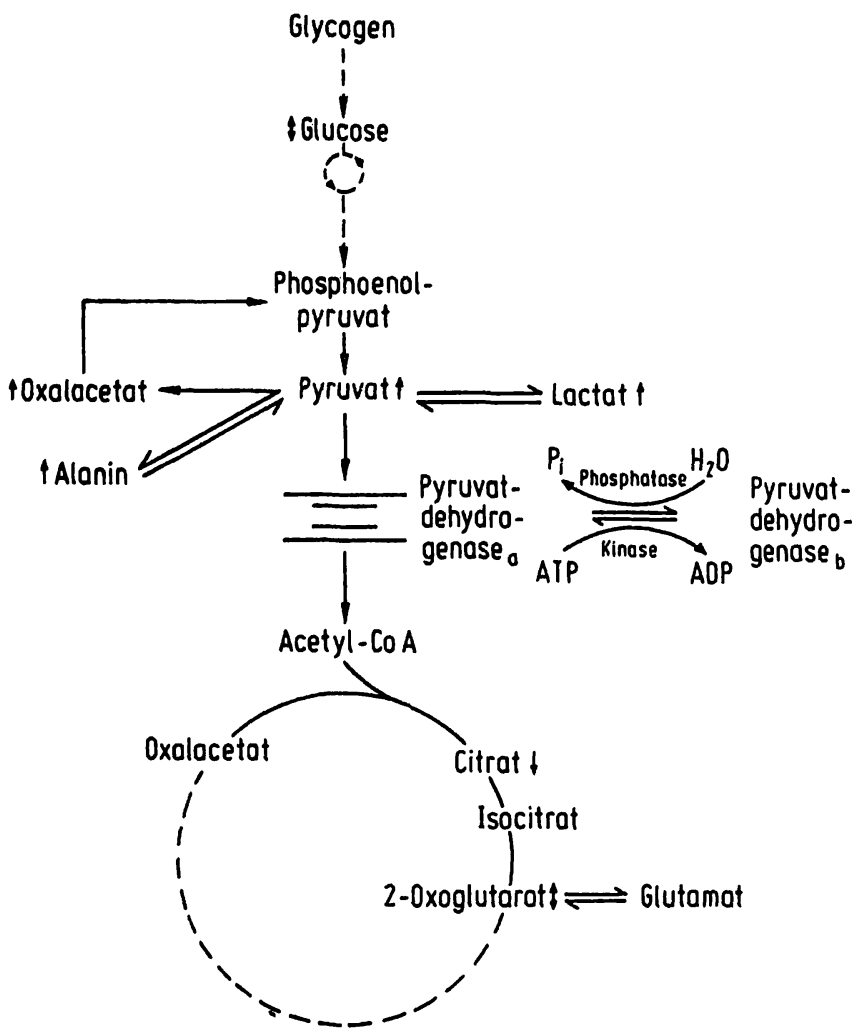

Schema 1. Vereinfachte Darstellung des Enzymdefektes und der Konsequenz einer defekten Pyruvatoxidation. $\uparrow$ Steigerung, $\downarrow$ Verminderung, $\uparrow$ normal.

Tab. 1 a. Klinisch-chemische Analysen des Kindes S. T. während Bettruhe und nach Arbeitsbelastung (20 Treppensteigen), verglichen mit Referenzwerten. n. b. = nicht bestimmt

\begin{tabular}{|c|c|c|c|}
\hline & Bettruhe & nach Belastung & Referenzwerte \\
\hline Lactat & $4,94 \mathrm{mmol} / \mathrm{l}$ & $15,20 \mathrm{mmol} / \mathrm{l}$ & $1,00-1,78 \mathrm{mmol} / \mathrm{l}$ \\
\hline Pyruvat & $172,00 \mu \mathrm{mol} / 1$ & $498,00 \mu \mathrm{mol} / \mathrm{l}$ & $41,00-67,00 \mu \mathrm{mol} / \mathrm{l}$ \\
\hline Oxalacetat & $1,45 \mathrm{mmol} / \mathrm{l}$ & n.b. & $0,06 \mathrm{mmol} / 1$ \\
\hline Citrat & $0,07 \mathrm{mmol} / \mathrm{l}$ & n.b. & $0,24-1,68 \mathrm{mmol} / \mathrm{l}$ \\
\hline Alanin & $485,30 \mu \mathrm{mol} / 1$ & n. b. & $99,00-313,00 \mu \mathrm{mol} / \mathrm{l}$ \\
\hline pH-Wert & 7,40 & 7,17 & $7,36-7,44$ \\
\hline Basenüberschuß & $-5,60 \mathrm{mmol} / \mathrm{l}$ & $-20,50 \mathrm{mmol} / \mathrm{l}$ & $2,50 \mathrm{mmol} / \mathrm{l}$ \\
\hline $\mathrm{pCO}_{2}$ arteriell & $27,10 \mathrm{~mm} \mathrm{Hg}$ & $19,10 \mathrm{~mm} \mathrm{Hg}$ & $34,00=46,00 \mathrm{~mm} \mathrm{Hg}$ \\
\hline Standardbicarbonat & $19,40 \mathrm{mmol} / \mathrm{l}$ & $9,40 \mathrm{mmol} / \mathrm{I}$ & $22,00-26,00 \mathrm{mmol} / \mathrm{l}$ \\
\hline $\mathrm{pO}_{2}$ arteriell & $45,00 \mathrm{~mm} \mathrm{Hg}$ & $50,60 \mathrm{~mm} \mathrm{Hg}$ & $74,40-108,0 \mathrm{~mm} \mathrm{Hg}$ \\
\hline
\end{tabular}

Tab.1b. Glucose (mmol/l) im Blutplasma des Kindes S.T. nach oraler Glucosebelastung und während eines Fastentests bei Bettruhe.

\begin{tabular}{llllll}
\hline & Nüchtern & $30 \mathrm{~min}$ & $60 \mathrm{~min}$ & $90 \mathrm{~min}$ & $120 \mathrm{~min}$ \\
\hline Orale Glucosebelastung & 5,49 & 5,53 & 4,86 & 4,75 & 4,64 \\
& Nüchtern & $2 \mathrm{~h}$ & $7 \mathrm{~h}$ & $12 \mathrm{~h}$ & \\
\cline { 2 - 6 } Fastentest & 4,42 & 4,61 & 5,23 & 4,79 \\
\hline
\end{tabular}


Die von uns gemessene katalytische Aktivität der Pyruvatcarboxylase in Leukocyten des Patienten S.T. entsprach den Werten gesunder Kontrollpersonen (S. T. $15,4 \mathrm{mU} / 10^{6}$ Leukocyten, Kontrolle A 12,0 $\mathrm{mU} / 10^{6}$ Leukocyten, Kontrolle B $18,0 \mathrm{mU} / 10^{6}$ Leukocyten). Das Fehlen einer Nüchternhypoglykämie zeigt eine intakte Gluconeogenese und ist ein weiteres Indiz für eine voll funktionstüchtige Pyruvatcarboxylase. Es wurde daher geprüft, ob die Oxidation des Pyruvats zu Acetyl-CoA blockiert ist. Abbildung 1 zeigt jedoch, daß die spontane Pyruvatdehydrogenase-Aktivität $\left(\mathrm{PDH}_{\mathrm{a}}\right)$ in Leukocyten beim ruhenden Patienten nicht signifikant vom Wert der gesunden Schwester abweicht.

Die Pyruvatdehydrogenase unterliegt einer Regulation durch Phosphorylierung und Dephosphorylierung. Es erscheint denkbar, daß - trotz scheinbar normaler Pyruvatdehydrogenase-Aktivität in der $\mathrm{Ru}$ hephase - die Aktivierbarkeit des Enzyms gehemmt ist, so daß es bei einem erhöhten Pyruvatangebot etwa bei motorischer Aktivität - zu einem Anstau von Pyruvat und dessen Metaboliten Lactat und Alanin kommt. Wie Abbildung 1 zeigt, führt motorische Belastung zu einem Anstieg der spontanen Aktivität von Pyruvatdehydrogenase bei einer gesunden Kontrolle, hat aber keine Effekte auf die Pyruvatdehydrogenase-Aktivität beim Patienten S. T.

In vitro wurde die Aktivierbarkeit der Pyruvatdehydrogenase in Leukozyten durch exogene Phosphoproteinphosphatase gemessen. Die Ergebnisse sind in Abbildung 2 dargestellt.

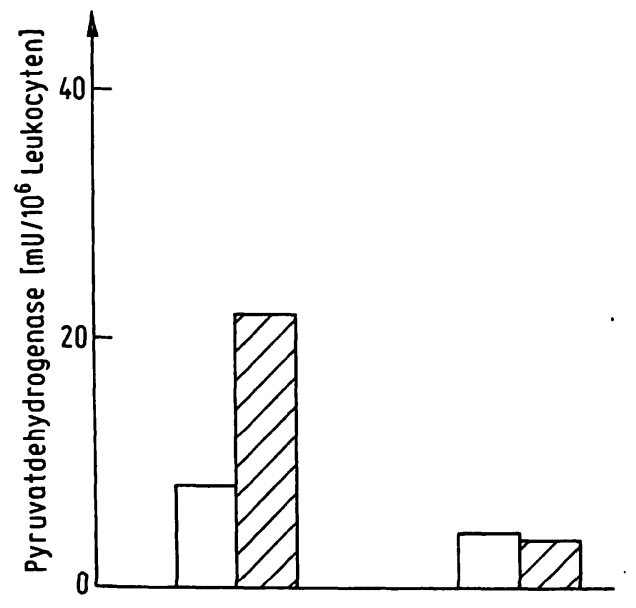

Abb. 1. Pyruvatdehydrogenase-Aktivität in Leukocyten des Patienten S.T. (rechts) und seiner gesunden Zwillingsschwester (links) in Ruhe ( $\square$ ) und nach Muskelarbeit $(\mathbb{Z})$. Dabei wurden 10 Minuten Bodenturnübungen durchgeführt und anschließend 30 Treppen gestiegen. Der Patient zeigte schon nach wenigen Minuten starke Ermüdungserscheinungen.
Die Inkubation des Zellhomogenates mit Phosphoproteinphosphatase stimuliert die Pyruvatdehydrogenase-Aktivität der Kontrollperson um mehr als das 10-fache, hingegen zeigt die Pyruvatdehydrogenase des Patienten nur eine geringfügige Aktivierung.

In Abbildung 3 werden die elektronenoptișchen Darstellungen des Muskelgewebes gezeigt. In erster Linie konnten Veränderungen im extrafibrillären Sarkoplasma nachgewiesen werden. Die Mitochondrien bilden breite Bänder zwischen êrhaltenen, regelrechten Myofibrillen. Ihre Zahl erscheint regionär etwas unterschiedlich, jedoch im allgemeinen stark vermehrt.

Es finden sich neben einigen normalen, stark vergröBerte und sehr unterschiedlich geformte Mitochondrien. Sie besitzen dicht gelagerte Cristae, meist ohne bevorzugte Orientierung. Im Sarkoplasma finden sich vermehrt Lipidtropfen, diê oft dicht von Mitochondrien umgeben sind.

Lichtmikroskopisch konnten keine Veränderungen an den Myofibrillen beobachtet werden, die vermehrten Mitochondrien ware auch hier zu erkennen (Abb. 4).

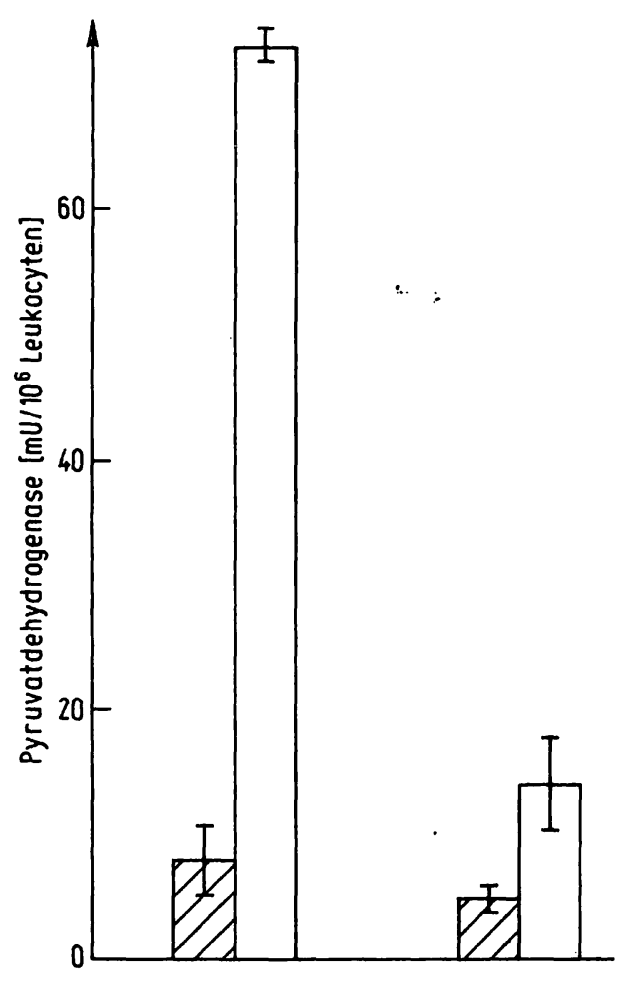

Abb. 2. Stimulierung ( $\square$ ) des Enzyms Pyruvatdehydrogenase in Leukocyten durch Zugabe von Phosphoproteinphosphatase $(50 \mu \mathrm{l}$ einer Suspension von $200 \mathrm{U} / \mathrm{mg}$ Protein zu $200 \mu$ l Leukocytenhomogenat) beim Patienten S.T. (rechts) und gesunden Kontrollen (links) bei motorischer Ruhe.

Mittel von fünf Versuchen \pm SD beim Patienten und sieben Versuchen \pm SD bei gesunden Kontrollen. 

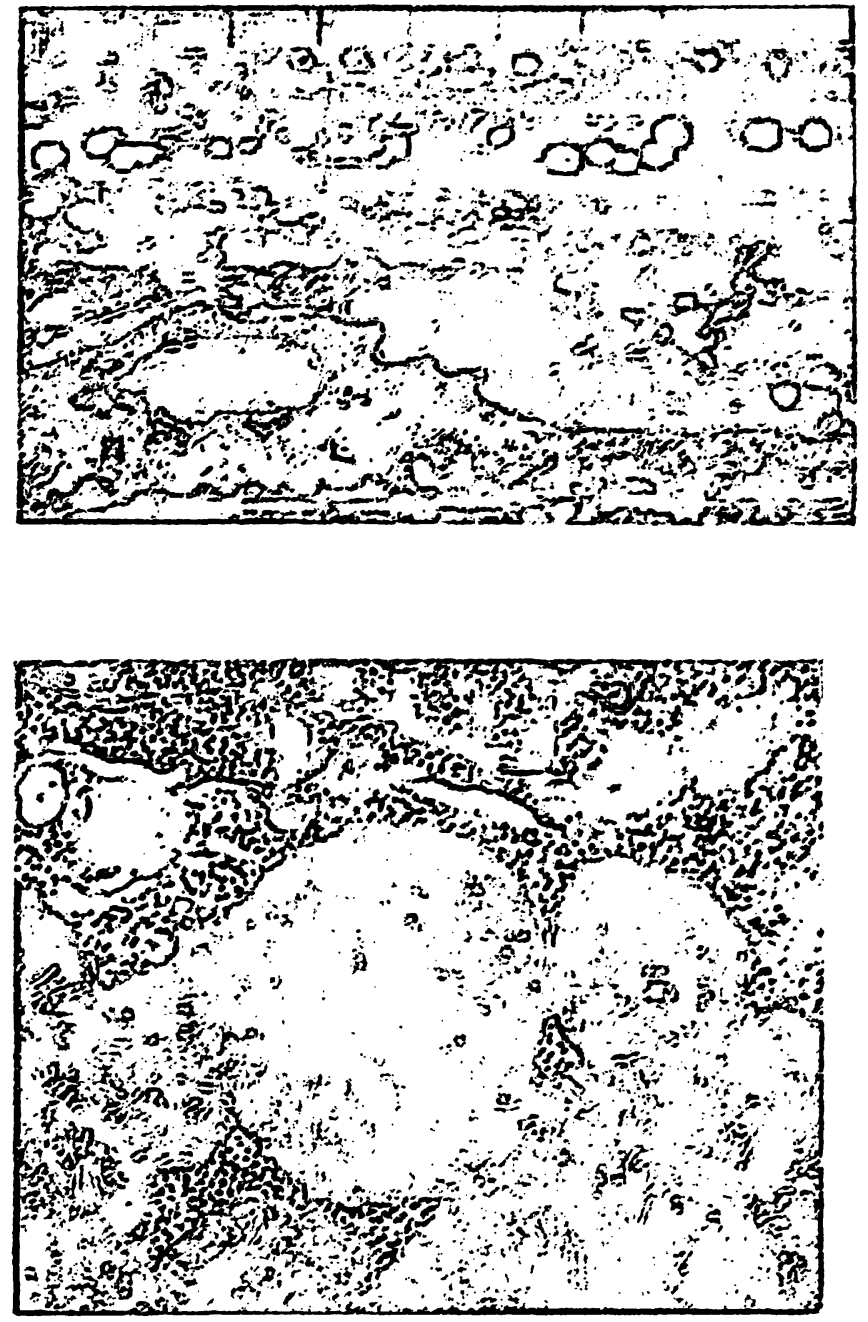

Abb. 3. Elektronenmikroskopische Darstellungen des intraoperativ entnommenen Muskelgewebes des M. quadriceps femoris bei motorischer Ruhe (Patient S. T.).

a) $\mathrm{EM} \times 2950$, zwischen erhaltenen Myofibrillen subsarkolemmal in Kernnähe große Ansammlung von Riesenmitochondrien.

b) $\mathrm{EM} \times 20000$, in Riesenmitochondrien dicht gepackte Cristae (Schnittführung annähernd senkrecht zur Längsachse).

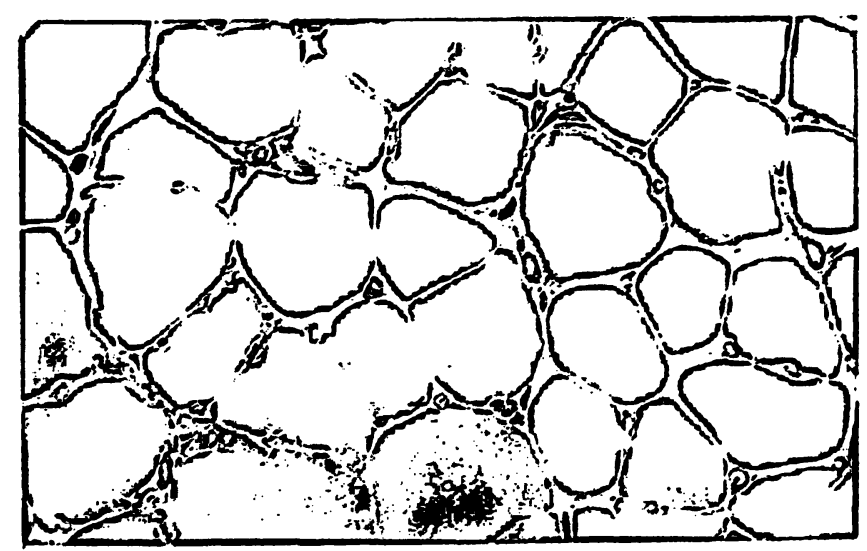

Abb. 4. Lichtmikroskopische Aufnahme eines Semidünnschnittes des intraoperativ entnommenen Muskelgewebes des M. quadriceps femoris bei motorischer Ruhe des $\mathrm{Pa}$ tienten S.T., Öl-Immersion, Leitz-Orthoplan/WildPhotoautomat MPS 45, Vergrößerung $38 \times$.

\section{Diskussion}

Eine gleichzeitige Erhöhung der Konzentration von Lactat und Pyruvat im Blut, wic im vorliegenden Fall, ist typisch für die "kindliche Lactatacidose“. Als Ursache der kindlichen Lactatacidose sind u.a. Defekte der Pyruvatcarboxylase $(33,34)$ und der Fructose-1,6-bisphosphatase beschrieben (35). Auch die Glykogenose vom Typ I führt infolge des Glucose6-phosphatasemangels zu einer Steigerung der Lactat- und Pyruvatkonzentration (36). Die genannten Enzymdefekte verursachen durch Hemmung der Gluconeogenese eine Nüchternhypoglykämie. Da im vorliegenden Fall der Nüchternblutzucker normal ist, scheiden die genannten Defekte als Ursache für das Krankheitsbild aus. Die in diesem Fall gemessenen erhöhten Konzentrationen von Lactat, Pyruvat und Alanin im Serum deuten somit auf eine Störung der Pyruvatoxidation hin.

Aufgrund der Stoffwechseldaten ist cine Verminderung der Pyruvatdehydrogenaseaktivität bei Fällen von kindlicher Lactatacidose mehrfach vermutet worden $(37,38,39)$. Ein Enzymdefekt ließ sich jedoch nur in wenigen Fällen nachweisen $(40,41)$. Von Blass et al. (42) wurde eine Verminderung, von Farell et al. (43) ein vollständiger Ausfall der Pyruvatdecarboxylaseaktivität bei Kindern mit kongenitaler Lactatacidose publiziert. Bei einem anderen Fall (44) war der Pyruvatdehydrogenase-Komplex durch einen Defekt der Lipoat-Acetyltransferase und/oder der Dihydrolipoat-Dehydrogenase insuffizient.

Bei den zitierten Beobachtungen wurde die spontane Pyruvatdehydrogenase Aktivität ohne Aktivierung durch Phosphoproteinphosphatase gemessen. Der hier beschriebene Fall unterscheidet sich von den oben zitierten dadurch, daß die spontane Pryruvatdehydrogenase-Aktivität nicht reduziert ist. Der Defekt der Pyruvatdehydrogenase zeigt sich jedoch in einer mangelnden Aktivierbarkeit durch Phosphoproteinphosphatase in vitro, bzw. nach motorischer Aktivität in vivo.

Eine Insuffizienz des Pyruvatdehydrogenasekomplexes als Folge eines Defektes der Enzymregulation wurde von Robinson \& Sherwood beschrieben. Die Autoren konnten nachweisen, daß bei dem von ihnen untersuchten Fall die Phosphoproteinphosphatase defizient ist. Dies scheidet jedoch bei dem hier vorliegenden Fall als Ursache des Krankheitsgeschehens aus. Bei Fehlen oder inaktiver Phosphoproteinphosphatase wäre eine maximale Aktivierung der Pyruvatdehydrogenase nach Zusatz von Phosphatase zu erwarten.

Für die von uns beobachtete verringerte Aktivierbarkeit der Pyruvatdehydrogenase kommen drei Ursachen in Betracht: 
a) Es liegt eine defekte Pyruvatdehydrogenase vor, deren Aktivität durch Phosphorylierung und Dephosphorylierung nicht beeinflußt wird und die somit der biologischen Regulation entzogen ist;

b) Es wird eine defekte Pyruvatdehydrogenase mit stark verminderter katalytischer Aktivität gebildet. Das Enzym ist prinzipiell noch durch Phosphorylierung und Dephosphorylierung aktivierbar, liegt in der Zelle jedoch bereits vollständig in der dephosphorylierten, aktiven Form vor, da das sich anstauende Pyruvat die Proteinkinase inhibiert. Darüberhinaus dürfte durch die ungenügende Pyruvatoxidation der Energiestoffwechsel so betroffen sein, daß der intramitochondriale ATP/ADP-Quotient niedrig ist und eine Aktivierung der Phosphoproteinphosphatase bewirkt;

c) Es wird eine normale Pyruvatdehydrogenase synthetisiert. Die Syntheserate ist jedoch stark vermindert, so daß der Gehalt der Zelle an Pyruvatdehydrogenase erniedrigt ist. Die relativ wenigen Enzymmoleküle sind durch die Erhöhung der Pyruvatkonzentration und wahrscheinlich auch durch den niedrigen ATP/ADP-Quotienten alle aktiviert und liegen ausschließlich in Form der $\mathrm{PDH}_{\mathrm{a}}$ vor.

In allen Fällen ist die Oxidation des Pyruvats $z u$ Acetyl-CoA so weit herabgesetzt, daß es zu einem Anstau von Pyruvat kommt. Die anstauende Brenztraubensäure wird teils zu Lactat reduziert, teils durch Transaminierung in Alanin übergeführt. Bedingungen, die physiologisch die Anflutung von Pyruvat steigern und die Pyruvatdehydrogenase aktivieren, wie z.B. motorische Aktivität, werden den Anstau von Pyruvat und u. a. auch von Lactat verstärken.

Eine Entscheidung darüber, ob die Enzymsynthese reduziert ist oder ob bei normaler Enzymmenge die katalytische Aktivität herabgesetzt ist, sollte prinzipiell durch eine Kombination von immunologischen Methoden mit einer Analyse der Enzymkinetik zu treffen sein und ist Gegenstand derzeitiger Untersuchungen.

Neben den bei einem Defekt der Pyruvatdehydrogenase zu erwartenden Änderungen der Pyruvat-, Lactat- und Alaninkonzentrationen wurde von uns noch eine Steigerung der Oxalacetatkonzentration und eine Erniedrigung der Citratkonzentration im Serum gemessen. Erhöhte Oxalacetatkonzentrationen sind bei kindlicher Lactatacidose auch von anderen Autoren beschrieben worden $(45,46)$. Die Ursache für die Oxalacetatakkumulation ist unklar. Möglich ist eine gesteigerte intramitochondriale Carboxylierung des sich anstauenden Pyruvats zu Oxalacetat, das sodann entweder via Aspartat oder über Malat in den extramitochondrialen Raum transportiert wird. Alternativ kommt eine Hemmung der Citrattsynthese in Betracht (vgl. Schema 1). Die letztgenannte Annahme wird durch die Erniedrigung der Citratkonzentration gestützt. Eine subnormale Citratkonzentration wurde auch von Strömme et al. (47) bei einem Fall von kongenitaler Lactatacidose beschrieben. Bei dem von Strömme et al. beschriebenen Fall war die Citratsynthaseaktivität im Vergleich zu Kontrollen unverändert. Die Autoren diskutierten die Erniedrigung der Citratkonzentration als Folge eines verminderten Angebotes von Acetyl-CoA durch die Pyruvatdehydrogenase. Diese Erklärung scheint uns jedoch angreifbar, da bei einem isolierten Pyruvatdehydrogenasedefekt ausreichend Acetyl-CoA aus dem Fettsäureabbau zur Verfügung stehen sollte. Wir halten es für wahrscheinlicher, daß die extramitochondriale Citratkonzentration durch Hemmung des Citratcarriers erniedrigt ist oder daß noch weitere Defekte der Mitochondrien vorliegen, die u. a. Enzyme der Atmungskette, des Citratzyklus und/oder der Fettsäureoxidation betreffen. Bei einer Erniedrigung der Aktivität der Atmungskette könnte auch über eine Ërhöhung der intramitochondrialen NADH-Konzentration eine Hemmung der Phosphoproteinphosphatase zu einer Verminderung der Pyruvatdehydrogenaseaktivität führen (48). Diese Möglichkeit scheint jedoch im vorliegenden Fall wenig wahrscheinlich, da bei einer Bestimmung der Enzymaktivität im $\mathrm{NAD}^{+}$-Überschuß gemessen wurde, und eine weitere Erhöhung der NAD ${ }^{+}$-Konzentration ohne Einfluß auf die Enzymaktivität blieb (Werte nicht publiziert). Das Vorliegen morphologisch alterierter Mitochondrien im vorliegenden Fall stütżt die Annahme, daß noch weitere mitochondriale Defekte vorliegen und gibt Anla $B$ zur Prüfung, ob die kongenitale Lactatacidose bei Pyruvatdehydrogenasedefizienz̃ nicht Ausdruck einer defekten Biogenese der Mitochondrien ist.

\section{Danksagung}

Herrn Prof. Dr. Otto Wieland, Diabetesforschungsinstitut, Krankenhaus München-Schwabing, wird für die hilfreiche Diskussion und für die Bereitstellung der Reagenzien zur Durchführung des Enzymtests gedankt. 


\section{Literatur}

1. Falk, R. E, Cederbaum, S.D., Blass, J.P., Gibson, G. E., Kark, R.A.P. \& Carrel, R.E. (1976) Pediatrics 58, 713-721.

2. Farrell, D. F., Clark, A. F., Scott, C. R. \& Wenneberg, R. P. (1975) Science 187, 1082-1084.

3. Wick, H., Schweizer, K. \& Baumgartner, R. (1977) Agents Actions 7, 405-410.

4. Papanastasiou, D., Lehnert, W., Schuchmann, L. \& Hommes, F. A. (1980) Helv. Pediatr. Acta 35, 253-260.

5. Atkin, B. M., Buist, N. R. M., Utter, M. F., Leiter, A. B. \& Banker, B. R. (1979) Pediatr. Res. 13, 109-116.

6. Skrede, S., Strömme, J. H., Stokke, O., Lie, S. O. \& Eldjarn, L. (1971) Acta Paediatr. Scand. 60, 138-145.

7. Hommes, F. A., Polman, H. A. \& Reering, J.D. (1968) Arch. Dis. Childhood 43, 423-426.

8. Rogers, J. G., Wilkinson, R. G., Skelton, I. \& Danks, D. M. (1981) J. Pediatr. 99, 272-273.

9. Robinson, B. H., Taylor, J. \& Sherwood, W. G. (1980) Pediatr. Res 14, 956-962.

10. Baker, L. \& Winegard, A. I. (1970) Clin. Res. 18, 281 - 288.

11. Pagliara, A. S., Karl, I. E., Keating, J., Brown, B. \& Kipnis, D. M. (1971) Clin. Res. 19, 481-488.

12. Israels, S., Haworth, J. C., Gourley, B. \& Ford, J. D. (1964) Pediatrics 34, 346-356.

13. Haworth, J.C., Ford, J.D. \& Younoszai, M. K. (1967) Can. Med. Ass. J. 97, 773-776.

14. Wendel, U., Przyrembel, H. \& Becker, K. (1978) Monatsschr. Kinderheilkd. 126, 140-147.

15. Strömme, J., Borud, O. \& Moe, J. P. (1976) Pediatr. Res. $10,60-66$.

16. Dennis, St. C. Padma, A., de Buysere, M. S. \& Olson, M. S. (1979) J. Biol. Chem. 254, 1252-1258.

17. Olson, M.S., Dennis, St. C., Routh, C. A. \& de Buysere, M.S. (1978) Arch. Biochem. Biophys. 187, 121-131.

18. Portenhauser, R., Wieland, O.H. \& Wenzel, H. (1977) Hoppe-Seyler's Z. Physiol. Chem. 358, 647-658.

19. Wieland, O. H., Patzelt, Ch. \& Löffler, G. (1972) Eur. J. Biochem. 26, 426-433.

20. Batenburg, J.J. \& Olson, M.S. (1976) J. Biol. Chem. 251, 1364-1370.

21. Robinson, B.H. \& Sherwood, W. G. (1975) Pediatr. Res. 9, 935-939.

22. Blass, J. P., Avigan, J. \& Uhlendorf, B. W. (1970) J. Clin. Invest. $49,423-432$.

23. Schweet, R.S. \& Cheslock, K. (1952) J. Biol. Chem. 199, $749-755$.

24. Scriba, P. \& Holzer, H. (1961) Biochem. Z. 334, 473-479.

25. Henning, G., Löffler, G. \& Wieland, O.H. (1975) FebsLetters $59,142-145$.
26. Wieland, O.H., Patzelt, Ch. \& Löffler, G. (1972) Eur. J. Biochem. 26, 426-433.

27. Siess, E. \& Wieland, O.H. (1972) Eur. J. Biochem. 26 , 96-105.

28. Cloc, R. \& Lamprecht, W. (1974) In: Methoden der enzymat. Analyse, (Bergmeyer, H. U., ed.) 3. Auflage, Bd. II, Verlag Chemie, Weinheim, 1491-1496.

29. Noll, F. (1974) In: Methoden der enzymat. Analyse, (Bergmeyer, H. U., ed.) 3. Auflage, Bd. II, Verlag Chemie, Weinheim, $1521-1525$.

30. Dagley, St. (1974) In: Methoden der enzymat. Analyse, (Bergmeyer, H. U., ed.) 3. Auflage, Bd. II, Verlag Chemie, Weinheim, 1607-1611.

31. Hohorst, H. J., Kreutz, F. H. \& Reim, M. (1974) In: Methoden der enzymat. Analyse, (Bergmeyer, H.U., ed.) 3. Auflage, Bd. II, Verlag Chemie, Weinheim, 1651-1652

32. Hommes, F. A., Polman, H.A. \& Reerink, J.D. (1968) Arch. Dis. Childhood 43, 423-426.

33. Brunette, M. G., Delvin, E., Hazel, B. \& Scriver, C. R. (1972) Pediatrics 50, 702-711.

34. Yoshida, T., Tada, T., Konno, T. \& Arakawa, T. (1971) Tok. J. Exp. Med. 99, 121-128.

35. Bakker, H.D., de Bree, P.K., Ketting, D., van Sprang, F. J. \& Wadman, S. K. (1974) Clin. Chim. Acta 55, 41-47.

36. Sadeghi-Nejad, A., Presente, E., Binkiewicz, A. \& Senior, B. (1974) J. Pediat. 85, 49-54.

37. Greene, H. J., Schubert, W. K. \& Hug, G. (1970) J. Pediat. $76,853-860$.

38. Gauthier, E. (1969) Helv. Med. Acta 35, 423-427.

39. Israels, S., Haworth, J. C., Gourley, B. \& Ford, J. D. (1964) Pediatrics 34, 346-353.

40. Papanastasiou, D., Lehnert, W., Schuchmann, L. \& Hommes, F. A. (1980) Helv. Pediatr. Acta 35, 253-260.

41. Wendel, U., Przyrembel, H. \& Becker, K. (1978) Monatsschr. Kinderheilkd. 126, 140-147.

42. Blass, J. P., Schulman, J. D., Young, D. S. \& Hom, E. (1972) J. Clin. Invest. 51, 1845-1850.

43. Farrell, D. F., Clark, A. F., Scott, C. R. \& Wenneberg, R. P. (1975) Science 187, $1082-1084$.

44. Robinson, B. H., Taylor, J., Kahler, S. G. \& Kirkham, H. (1981) Eur. J. Pediatr. 136, 35-39.

45. Greene, H. J., Schubert, W. K. \& Hug, G. (1970) J. Pediatr. $76,853-860$.

46. Schärer, K., Marty, A. \& Mühlenthaler, J. P. (1968) Pediat. Acta 23, 107-114.

47. Strömme, J. H., Borud, O. \& Moe, J. P. (1976) Pediat. Res. 10, 60-66.

48. Pettit, F.H., Pelley, J.W. \& Reed, L. J. (1975) Biochem. Biophys. Res. Comm. 65, 575-582.

\section{Dr. Erika Dworzak Institut für Medizinische Chemie und Biochemie Universität Innsbruck Fritz-Preglstraße 3/5 A-6020 Innsbruck}


